\title{
Special issue of IREC2016 conference selected papers
}

\author{
Mohammad Pourgol-Mohammad ${ }^{1}$ Alireza Ahmadi ${ }^{2}$
}

Published online: 22 August 2017

(C) The Society for Reliability Engineering, Quality and Operations Management (SREQOM), India and The Division of Operation and Maintenance, Lulea University of Technology, Sweden 2017

This issue consists of a set of updated papers that were presented at the 4th International Reliability Engineering Conference (IREC2016) held in Tabriz, Iran, on May 2-4, 2016. The conference is held twice-yearly to provide a pertinent atmosphere for the scientific presentation and discussion of the multi-disciplinary researches on reliability engineering and risk analysis for industries, and academic communities.

On the late 2000s in the Tehran, the IREC Conference grew out of the recognition that technical forum was needed to exchange ideas related to reliability and risk methods. The three previous conferences were held on (1) IREC 2010 by Iranian Aerospace Research Institute in Tehran, (2) IREC2012 by Iranian Aerospace Research Institute in Tehran, (3) IREC2014 by Amir-Kabir University of Technology in Tehran. The success of these early conferences led to a realization that a series of conferences was needed. The objective of these conferences is to promote rational decision making to assure safety and reliability and to optimize the use of resources for complex systems. IREC2016 provided this platform for researchers and engineers to meet, discuss and share their findings and experiences. The conference covered scientific, technical, and societal aspects of risk, safety and reliability, and their application to multidisciplinary areas with a special focus on critical mission systems like aviation, space and nuclear industry.

\footnotetext{
Mohammad Pourgol-Mohammad

pourgolmohammad@sut.ac.ir

Sahand University of Technology, Tabriz, Iran

2 Lulea University of Technology, Luleå, Sweden
}

Out of the 82 papers presented at IREC2016, a total of 13 were finally selected by the Technical Program Committee for publication in this special issue of International Journal of System Assurance Engineering and Management. These papers were expanded and then underwent a full peer review process for publication. While any small subset of papers selected from IREC2016 conference cannot cover all of the technical areas or special sessions addressed during the conference, these papers do provide insights into the types of reliability engineering and risk analysis areas that make the IREC conferences popular with researchers and practitioners in the fields of probabilistic safety, maintainability, reliability, risk, and management.

The first paper entitled "Reliability Analysis of MultiState Emergency Detection System Using Simulation Approach Based on Fuzzy Failure Rate" deals with the reliability of a multistate system i.e. Launch Emergency Detection System with unknown data owning to events, based on FTA and fuzzy failure rates. Using fuzzy arithmetic, events time-to-failure are generated and top event time to failure is calculated. The results are compared with the analytical solution and shows that this method has more accuracy and suitable for all real industrial and complex systems in spite of less effort and time consuming.

The second paper with title of "Evaluating MTTF of 2-out-of-3 Redundant Systems with Common Cause Failure and Load Share Based on Alpha Factor and Capacity Flow Models" presents three models to evaluate MTTF and MTBF of a 2-out-of-3 redundant system with dependent failures and repair. Model I is potentially used to evaluate MTTF of a 2-out-of-3 redundant system with CCF. The MTTF of 2-out-of-3 redundant systems with $\mathrm{CCF}$ and load share is evaluated using Model II. If the 
components of a redundant system are repairable, Model III can be used to evaluate MTBF.

The third paper entitled "Estimating the Change Points of Bathtub-Shaped Hazard Functions" studies life time intervals for bathtub shaped hazard function. First change point is calculated by a combination of two criteria of minimum of hazard function and the maximum change in decreasing slope of hazard function. Second change point is calculated by determination of maximum change in increasing slope of hazard function after first change point. The exact estimation of change point assists to plan appropriate burn-in, guarantee, maintenance, repair and replacement strategies. The Bayesian inference is used for parameter estimation.

The fourth paper with title of "Reliable Flight Computer for Sounding Rocket with Dual Redundancy: Design and Implementation Based on COTS Parts" presents the design and implementation of a dual-redundant reliable flight computer with limited reliability data. One of the main and substantial parts of the sounding rockets is the flight computer with expected high reliability. The procurement limitation of the special-purpose space-grade components, motived this research to take a component-level dual-redundant architecture by using commercial off-the-shelf (COTS) components. Analytical results show that the reliability of a dual-redundant computer based on COTS parts is comparable with the reliability of a single computer based on special-purpose parts with the order of magnitude lower failure rates.

The fifth paper entitled "Design for Reliability of Automotive Systems; Case Study of Dry Friction Clutch" proposes an integrated methodology based on design for reliability of automotive systems and considering its reliability/safety critical sub-systems. The system is modeled by RBD method, simulated by Monte Carlo technique. The results of FMEA and reliability evaluation are used for system improvement by reducing the components' failure rates and potential change of system configuration. As a case study, dry friction clutch is studied for assessment of the proposed method.

The sixth paper with title of "Risk Assessment of Sensor Failures in a Condition Monitoring Process; DegradationBased Failure Probability Determination" proposes a riskbased measure to take into account the consequence of sensor missed-detection in system health monitoring. First, a sensor characteristic is selected through which the degradation process is affected. Then a degradation model is developed to calculate the sensor time to failure. The consequences of missed-detection are also corresponding quantifiable potential losses through both failure costs and maintenance expenditure. Finally, all feasible sensors placement scenarios are compared due to proposed risk measure.
The seventh paper with title of "Gas Turbine Preventive Maintenance Optimization Using Genetic Algorithm" proposes an intelligent maintenance optimization tool based on genetic algorithm. The proposed algorithm would make the balance between maintenance costs and down time cost while maintaining system availability on a predefined level. Besides, maintenance constraints are considered. The algorithm is applied on Siemens SGT600 gas turbine in which the total cost is reduced more than $80 \%$ while availability is improved roughly $2 \%$.

The eighth paper with title of "Risk Based Maintenance Strategy: A Quantitative Approach Based on Time to Failure Model" argues that an appropriate statistical model should be selected to predict the reliability of the system based on the nature of historical failure. Thereafter, based on the defined reliability characteristics of the system, an appropriate maintenance strategy needs to be established. In this study, a systematic methodology is developed, based on the available guidelines for maintenance strategy selection, founded on the reliability analysis of failure data from the crushing and mixing bed hall unit at a cement factory.

The ninth paper entitled "Effects of Shutdown Period Extension on Core Damage Frequency" proposes a comprehensive low power and shutdown (LPSD) probabilistic risk assessment modeling for a typical nuclear power plant. It results in 2.27E-06/year, for an LPSD CDF of representative refueling shutdown. Upon varying the POS duration, the contribution of $\mathrm{CDF}$ of the two most dominant POSs changes from 66.24 and $10.45 \%$, to 70.20 and $1.99 \%$, respectively. Besides, percent changes in CDF of POS 7 and 5 whose durations undergo -4.76 and $3.31 \%$, respectively, are -83.38 and $65.5 \%$. The results are useful for outage planning and shutdown management.

The tenth paper with title of "Probabilistic Analysis of Containment Structural Performance in Severe Accidents" reviews thoroughly the methodology of structural fragility analysis for pressurization. Its practical application is demonstrated on a real case PWR containment. The reactor containment is analyzed using a finite element method, and the ultimate pressure is computed. Containment performance is further analyzed using the structural fragility method.The final outcome from this study is the overpressure fragility curve, depicting the containment probability of failure at a given pressure.

The eleventh paper with title of "Evaluating ThermoHydraulics Uncertainties of Success Criteria in Probabilistic Risk Assessment" evaluates, through a systematic framework, thermo-hydraulics uncertainties of success criteria in probabilistic risk assessment for nuclear power plants which is not formally performed in industrial practice. An application of the methodology is demonstrated for 
loss of coolant accident in a PWR type nuclear power plant.

The twelfth paper with title of "Identification of Plastic Properties of Metallic Structures by Artificial Neural Networks based on Plane Strain Small Punch Test" discusses a novel approach, the plane strain small punch test (SPT), to assess the strength of in-service components. To do so, a new apparatus was designed and manufactured to perform a series of plane strain SPT in room temperature. An artificial neural network was established and trained by the corresponding load displacement responses obtained from the simulations to predict the plastic properties of Stainless Steel 304L.

The last paper entitled "Brain Tumor Growth Simulation: Model Validation through Uncertainty Quantification" analyzes an image-based tumor growth model by taking into account uncertainties in the model parameters by using Monte Carlo simulation. The time needed for tumor to grow to its fatal size was estimated. Comparison of the predicted time distribution in case of uncertainties with clinical-based report shows a decrease in dispersity of predicted data that highlights the importance of uncertainty analysis. Also, the wide range for survival time shows the importance of choosing proper parameters by clinical data. Recommendations were made for increasing the validity of the models.

We would like to thank all of the conference authors and presenters. Additional thanks go out to the volunteers on the Technical Program Committee whose efforts make these types of conferences possible. Lastly, we would like to thank the Honorary Chair, Dr. Ali Mosleh, for all his encouragement and supports. We would like to acknowledge efforts of the members of the executive committee especially Dr. Mehdi Mirzaei and Dr. Mojtaba Yazdani, the executive chairs, for fantastic organization of the conference. The editors appreciate the timely efforts of the journal staff to follow up the required actions and make this special issue happen. 